\title{
Typical Finding of Mechanical Obstruction Suggested in High-resolution Manometry
}

\author{
Hyung Hun Kim, Moo In Park, ${ }^{*}$ Seun Ja Park and Won Moon \\ Department of Internal Medicine, Kosin University College of Medicine, Busan, Korea
}

A 66-year-old man presented to our motility clinic with a complaint of difficulty swallowing solid food and liquid that had persisted for the past 2 months. His symptoms were intermittent and did not seem progressive. Esophagoscopy revealed a very tightly closed esophagogastric junction (EGJ) (Fig. 1A). We were unable to pass an endoscope through the EGJ. Fluoroscopy revealed a bird-beak like narrowing of the distal esophagus at the EGJ (Fig. 1B). High-resolution manometry (HRM) revealed an elevated mean integrated relaxation pressure of $29.9 \mathrm{mmHg}$, and simultaneous contractions were observed in 7 out of 10 swal- lows (Fig. 2). No abnormalities were noted on a multiple swallow test. A biopsy from the EGJ indicated moderately differentiated adenocarcinoma, and positron emission tomography CT scans revealed intense hypermetabolic status at the EGJ and cardia of the stomach.

Following the Chicago classification (2011), we categorized our findings as EGJ outflow obstruction due to elevated integrated relaxation pressure and instances of intact peristalsis. ${ }^{1}$ We considered 2 possible types of causal disease, variant achalasia and mechanical obstruction. ${ }^{2}$ We were unable to pass the endo-
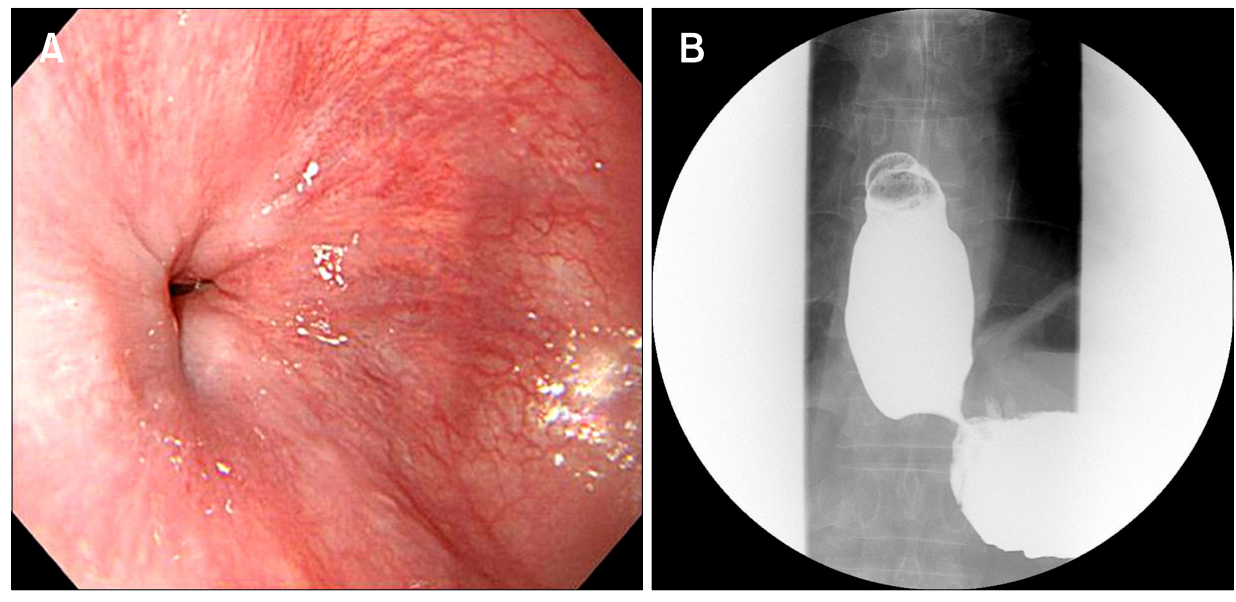

Figure 1. Basic examinations prior to high-resolution manometry. (A) Esophagoscopy revealed a tightly narrowed esophagogastric junction (EGJ). The endoscope was not able to pass the EGJ. (B) Fluoroscopic examination revealed a bird-beak like, abrupt tapering of the distal esophagus and EGJ.

Received: April 11, 2012 Revised: April 23, 2012 Accepted: April 29, 2012

(c) This is an Open Access article distributed under the terms of the Creative Commons Attribution Non-Commercial License (http://creativecommons. org/licenses/by-nc/3.0) which permits unrestricted non-commercial use, distribution, and reproduction in any medium, provided the original work is properly cited.

*Correspondence: Moo In Park, MD

Department of Internal Medicine, Kosin University College of Medicine, Gamcheon-ro 262, Seo-gu, Busan 602-702, Korea

Tel: +82-51-990-5205, Fax: +82-51-990-5055, E-mail: mipark@ns.kosinmed.or.kr

Financial support: None.

Conflicts of interest: None. 

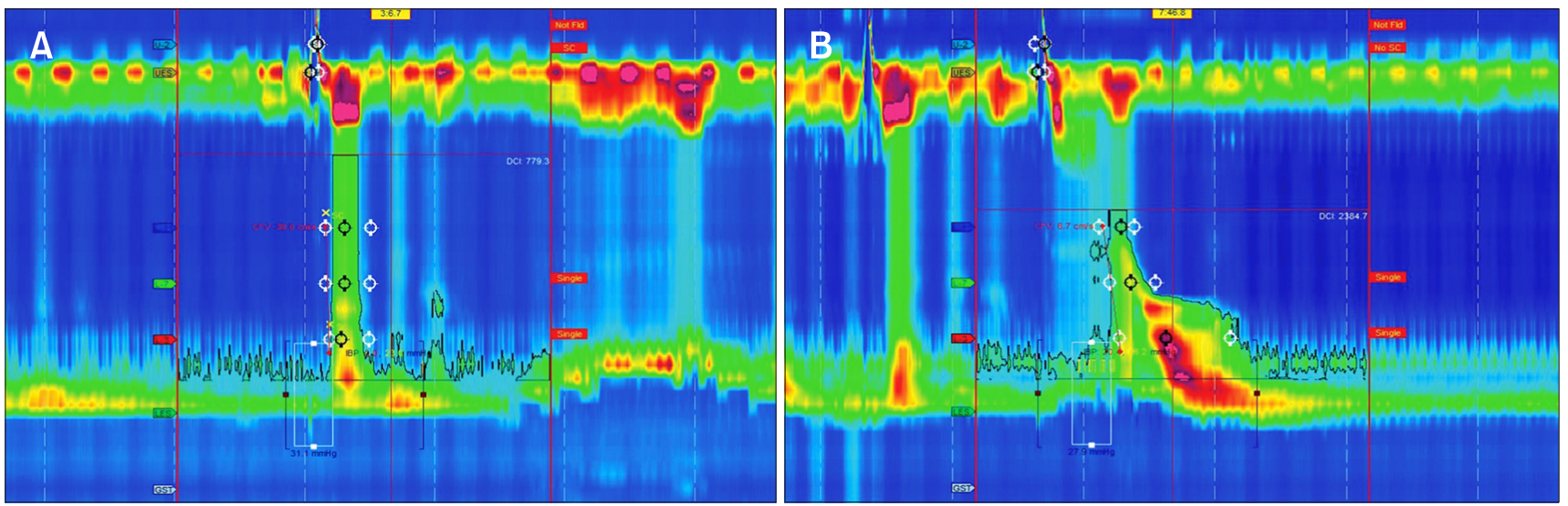

Figure 2. High-resolution manometry (HRM) spatio-temporal plots. (A) HRM revealed elevated integrated relaxation pressure $(31.1 \mathrm{mmHg})$ with increased contractile front velocity $(39.0 \mathrm{~cm} / \mathrm{sec})$. (B) HRM also showed increased integrated relaxation pressure $(27.9 \mathrm{mmHg})$ and normal peristaltic contraction and contractile front velocity $(6.7 \mathrm{~cm} / \mathrm{sec})$.

scope beyond the EGJ, so we suspected a mechanical obstruction rather than variant achalasia. To differentiate between the 2 conditions, we performed biopsies and positron emission tomography CT scans, and found that our patient's symptoms were caused by adenocarcinoma located at the cardia. This case showed the typical HRM findings of mechanical EGJ outflow obstruction, which resembled the pattern of EGJ flow obstruction if it was a primary esophageal motility disorder, by the 2011 Chicago classification. ${ }^{1}$

\section{References}

1. Bredenoord AJ, Fox M, Kahrilas PJ, et al. Chicago classification criteria of esophageal motility disorders defined in high resolution esophageal pressure topography. Neurogastroenterol Motil 2012; 24(suppl 1):57-65.

2. Kim HH, Park MI, Baik JH, Park SJ, Moon W. Variant achalasia: a new category of the chicago classification published in 2011. J Neurogastroenterol Motil 2012;18:102-103. 